\title{
An alternative NMSSM phenomenology with manifest perturbative unification
}

\author{
Riccardo Barbieri ${ }^{a}$, Lawrence J. Hall ${ }^{b}$, Anastasios Y. Papaioannou ${ }^{b}$, \\ Duccio Pappadopulo $^{a}$ and Vyacheslav S. Rychkov ${ }^{a}$ \\ a Scuola Normale Superiore and INFN, Piazza dei Cavalieri 7, I-56126 Pisa, Italy \\ ${ }^{b}$ Department of Physics, University of California, Berkeley, and \\ Theoretical Physics Group, LBNL, Berkeley, CA 94720, USA
}

\begin{abstract}
Can supersymmetric models with a moderate stop mass be made consistent with the negative Higgs boson searches at LEP, while keeping perturbative unification manifest? The NMSSM achieves this rather easily, but only if extra matter multiplets filling complete $S U(5)$ representations are present at intermediate energies. As a concrete example which makes use of this feature, we give an analytic description of the phenomenology of a constrained NMSSM close to a Peccei-Quinn symmetry point. The related pseudo-Goldstone boson appears in decays of the Higgs bosons and possibly of the lightest neutralino, and itself decays into $b \bar{b}$ and $\tau \bar{\tau}$.
\end{abstract}




\section{Introduction and motivations}

The absence of any clear signal of the Higgs boson(s) at LEP is a disturbing fact for the Minimal Supersymmetric Standard Model (MSSM). As well known a heavy stop could be the explanation. Although possible, however, this weakens the view that requires supersymmetry to be visible at the LHC, especially since the top, and the stop, have the strongest coupling to the Higgs boson system. In the attempt to avoid this quite unpleasant road, several proposals have in fact been made: among them, the consideration of the Next to Minimal Supersymmetric Standard Model (NMSSM) has received a great deal of attention. It is in fact true that the extra contribution to the quartic Higgs coupling arising in the NMSSM can easily accommodate a lightest Higgs boson even much heavier than in the MSSM[1],2]. A strong constraint, however, to which we stick in this paper, is its compatibility with manifest perturbative unification.

Although the NMSSM is a very minimal extension of the MSSM, it has a drawback: it allows to introduce several more parameters, which often make the various analyses difficult to follow or can even obscure the very search for significant phenomenological patterns. In this paper we try to clarify a possibility offered by the NMSSM to comply with the LEP constraint:1 in a weakly finetuned and not too narrow region of its parameter space, while insisting on a relatively light stop. A key point is that such possibility rests on the largest possible values of the usual $\lambda S H_{1} H_{2}$ coupling of the NMSSM consistent with manifest perturbative unification, including the possible existence of extra matter multiplets filling complete $S U(5)$ representations at intermediate energies 8 ].

With CP conserved in the scalar sector, the NMSSM has three CP even and two CP odd neutral fields. With the standard definition of the Higgs doublets $H_{1}$ and $H_{2}$, the only scalar with tree level coupling to the vector boson pairs VV, often called $h$ since it is the closest to the Standard Model (SM) Higgs boson, has the composition ${ }^{2}$

$$
h=h_{1}^{0} \cos \beta+h_{2}^{0} \sin \beta
$$

and tree-level diagonal mass-squared

$$
\left(m_{h}^{0}\right)^{2}=M_{Z}^{2} \cos ^{2} 2 \beta+\lambda^{2} v^{2} \sin ^{2} 2 \beta,
$$

corrected by the well known radiative contribution from top-stop loops (for moderate mixing, $\left.\left|A_{t} / m_{\tilde{t}}\right| \lesssim 1\right)$

$$
m_{h}^{2} \simeq\left(m_{h}^{0}\right)^{2}+\frac{3 m_{t}^{4}}{4 \pi^{2} v^{2}} \log \frac{m_{\tilde{t}}^{2}}{m_{t}^{2}}
$$

We do not show in this radiative correction a small positive contribution due to $A_{t}$, because in practice for moderate mixing it is compensated by a further negative $O\left(\alpha_{s} \alpha_{t}\right)$ correction at the two-loop order. Thus the one-loop result (1.3) remains a reasonable approximation.

Note that equation $(1.2)$ is valid for any scalar potential of the form

$$
V=V^{\text {gauge }}\left(H_{1}, H_{2}\right)+\mu_{1}^{2}(S)\left|H_{1}\right|^{2}+\mu_{2}^{2}(S)\left|H_{1}\right|^{2}-\left(\mu_{3}^{2}(S) H_{1} H_{2}+\text { h.c. }\right)+\lambda^{2}\left|H_{1} H_{2}\right|^{2}+V(S)
$$

\footnotetext{
${ }^{1}$ For other attempts see e.g. 3, 4, [5. There are, however, two different aspects of the problem that may have not been equally addressed in these works: the level of fine-tuning in the $Z$-mass and the narrowness of the region of parameter space consistent with current data.

${ }^{2}$ As usual, $\tan \beta=v_{2} / v_{1}, v_{i}=\left\langle H_{i}^{0}\right\rangle, v_{1}^{2}+v_{2}^{2}=174 \mathrm{GeV}$.
} 


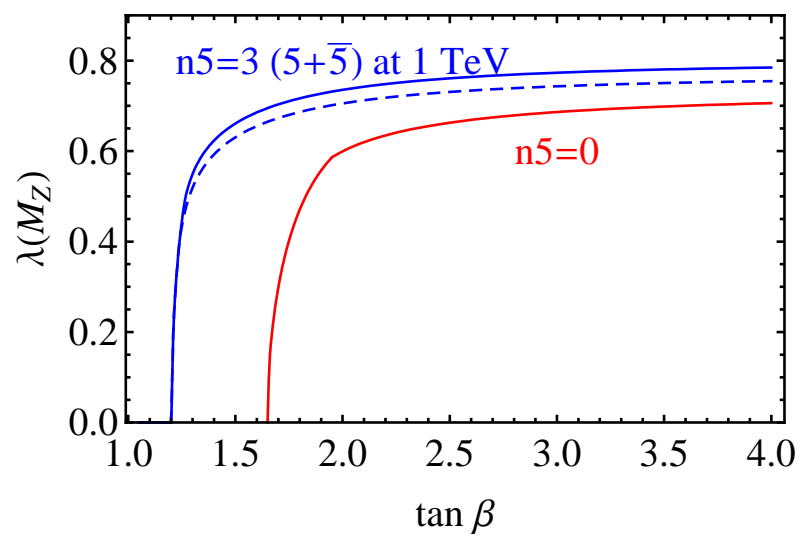

Figure 1: Lower (red) curve: the maximal value of $\lambda\left(M_{Z}\right)$ as a function of $\tan \beta$ in NMSSM without extra matter at intermediate energies, subject to the condition $\lambda_{\text {GUT }} / 4 \pi<0.3$, $\kappa_{\mathrm{GUT}}=0$. Upper (blue) curves: same but with $n_{5}=3$ extra $5+\overline{5}$ at $1 \mathrm{TeV}$, and $\lambda_{\mathrm{GUT}} / 4 \pi<$ $0.3,0.15$.

i.e., in particular, for any NMSSM which stays perturbative up to the GUT scale.

The phenomenology of the NMSSM in relation with the Higgs boson searches at LEP certainly depends on the value of $m_{h}$, but crucially also on the mixings of $h$ with the two other CP even scalars, since $m_{h}^{2}$, being a diagonal entry of a positive definite squared mass matrix, gives only an upper bound on the mass squared of the lightest physical CP-even scalar.

With this in mind, this paper consists of two logically independent but also complementary parts. In the first one we discuss the maximum possible values of the coupling $\lambda$, and therefore of $m_{h}$, in presence of extra matter multiplets filling complete $S U(5)$ representations at intermediate energies (Sect. 2). Furthermore, based on the values that we find for $m_{h}$, we consider a simple and generic $2 \times 2$ mixing model between $h$ and the lightest among the two remaining CP even scalars, which, before mixing with $h$, do not couple at all with VV (Sect. 3). In the second part we describe a fully detailed and motivated version of the NMSSM with an approximate Peccei-Quinn symmetry that realizes the phenomenological pattern outlined in the first part. This approximate symmetry restricts the number of effective parameters and makes possible an analytic description of most of the relevant features we want to underline.

\section{On the maximal value of the $S H_{1} H_{2}$ coupling}

From eq. 1.2 $m_{h}$ is especially sensitive to the value of the coupling $\lambda$ at the weak scale, which is constrained by demanding that $\lambda$ stays perturbative in its RGE evolution all the way up to the GUT scale. More specifically, since $\lambda$ grows with the energy from the weak to the GUT scale, we require for its value at the GUT scale, $\lambda_{G U T}<0.3 \cdot 4 \pi$.

The RGEs of $\lambda$ and of other relevant couplings can be found in Appendix A. A significant indirect effect on the evolution of $\lambda$ is caused by the presence at intermediate energies of vectorlike supermultiplets filling complete $S U(5)$ representations [8]. These multiplets increase the gauge couplings at higher energies, which in turn slows down the growth of both $\lambda$ and $y_{t}$, delaying the 


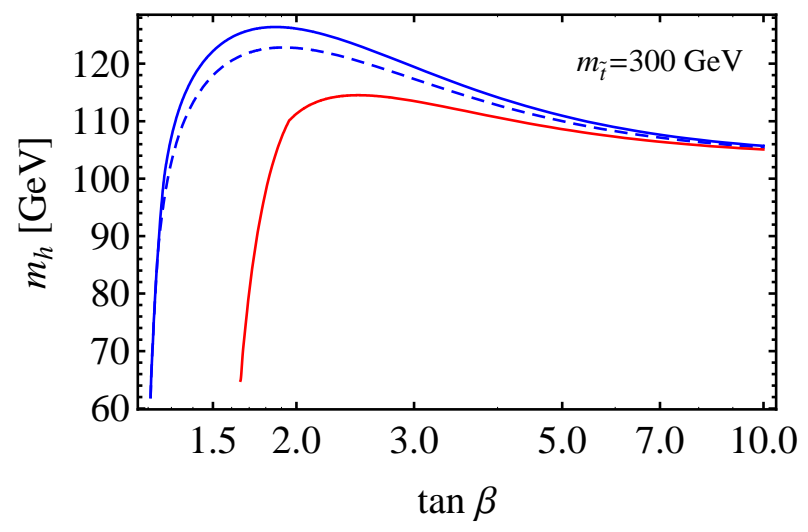

Figure 2: The maximal value of $m_{h}$, see Eq. 1.3), in NMSSM without extra matter (red) and with $n_{5}=3$ extra $5+\overline{5}$ at $1 \mathrm{TeV}$ (blue). The values of $\lambda_{\text {GUT }}$ are the same as in Fig. 1 . The stop mass is fixed at $m_{\tilde{t}}=300 \mathrm{GeV}$ with moderate mixing, $\left|A_{t} / m_{\tilde{t}}\right| \lesssim 1$.

onset of nonperturbative behavior. This effect is illustrated in Fig. 1 which shows as function of $\tan \beta$ the maximum value of $\lambda$ at the weak scale without or with extra-matter effects (three $(5+\overline{5})$ of $S U(5)$ at the weak scale), for the current value of $m_{t}=171 \mathrm{GeV}{ }^{3}$ Consequently, from eq.s 1.2 , 1.3), Fig. 2 gives the maximum value of $m_{h}$ for a moderate stop mass, $m_{\tilde{t}}=300 \mathrm{GeV}$. The upper blue curves, for $\lambda_{\mathrm{GUT}} / 4 \pi=0.3,0.15$ are again with three $(5+\overline{5})$ of $S U(5)$ at the Fermi scale, whereas the lower red curve, for $\lambda_{\mathrm{GUT}} / 4 \pi=0.3$, includes in the RGE evolution the standard matter effects only.

Several features in these figures are worth being observed. All the curves in Fig. 2 go for large $\tan \beta$ to a common asymptotic value which is the upper bound on $m_{h}$ in the MSSM (for the same $m_{\tilde{t}}=300 \mathrm{GeV}$ and moderate mixing). Relative to this value, the increment in $m_{h}$ due to the extra three $(5+\overline{5})$ is clearly significant, especially since without extra matter the maximum value of $m_{h}$ barely touches the LEP bound on the SM Higgs boson mass of about $115 \mathrm{GeV}$. This is even more so since the upper limit on $m_{h}$ is essentially saturated for wide variations of $\lambda_{\mathrm{GUT}}$, in its upper range, as shown by the close upper curves in Fig. 2.

Both Fig. 1 and 2 are for vanishing $\frac{\kappa}{3} S^{3}$ coupling in the superpotential, but they are all insensitive to any choice of $\kappa_{\mathrm{GUT}} / 4 \pi \leq 0.05$, since $\kappa$ is rapidly driven to zero at lower energies by the RGE evolution. A larger $\kappa_{\mathrm{GUT}}$ would however reduce the maximum $\lambda$ at the weak scale.

Finally notice that the curves without any extra matter start at $\tan \beta \simeq 1.6$ because at lower $\tan \beta$, unlike in the case with extra matter, the top Yukava coupling hits by itself the perturbative bound of $0.3 \cdot 4 \pi$ before getting to the unification scale.

Recently, Dine, Seiberg and Thomas [10] have claimed that in a singlet extension of MSSM based on the superpotential $\mu H_{1} H_{2}+\lambda S H_{1} H_{2}+\frac{1}{2} M S^{2}$ one can raise the Higgs mass by a significant amount while maintaining manifest perturbative unification without extra matter at intermediate scales. E.g., one of their examples ([10], Sec. 4.1) had $m_{h}=120 \mathrm{GeV}$ for $\tan \beta=4, \lambda=0.7$,

\footnotetext{
${ }^{3}$ The example $n_{5}=4$ at $1 \mathrm{TeV}$ emphasized in [8] gives non-perturbative values of $\alpha_{\mathrm{GUT}}$ once the 2-loop terms are included in gauge beta functions.
} 


\begin{tabular}{|c|c||c|c|l|}
\hline \multicolumn{2}{|c||}{$n_{5}=0$} & \multicolumn{2}{c|}{$n_{5}=3$} & \\
\hline$\alpha_{S}\left(M_{Z}\right)$ & $\alpha_{G}$ & $\alpha_{S}\left(M_{Z}\right)$ & $\alpha_{G}$ & \\
\hline $\mathbf{0 . 1 1 7}$ & 0.041 & $\mathbf{0 . 1 1 7}$ & 0.103 & 1-loop \\
\hline $\mathbf{0 . 1 3 0}$ & 0.043 & $\mathbf{0 . 1 2 3}$ & 0.154 & 2-loop numerical \\
\hline $\mathbf{0 . 1 2 9}$ & 0.043 & $\mathbf{0 . 1 2 2}$ & 0.143 & 2-loop analytical \\
\hline
\end{tabular}

Table 1: Prediction for $\alpha_{S}\left(M_{Z}\right)$ in the standard case $\left(n_{5}=0\right)$ and for $n_{5}=3(5+\overline{5})$ at 1 $\mathrm{TeV}$. We use one and two loop gauge beta functions given in Appendix A without the two loop contributions of $\lambda$ and $y$, which can later be included perturbatively. The input $\overline{\mathrm{MS}}$ values are $\hat{\alpha}\left(M_{Z}\right)^{-1}=127.918, \sin ^{2} \hat{\theta}_{W}\left(M_{Z}\right)=0.23122$. We do not include any threshold corrections. The last line of the table is obtained by treating the 2-loop terms as perturbative corrections to the 1-loop results, following the standard method as described e.g. in [11].

$m_{\tilde{t}}=300 \mathrm{GeV}^{4}$ and no mixing, which is in clear contradiction with Fig. 2. As is stressed in the Introduction, our bound on $m_{h}$ applies to any Higgs potential of the form (1.4), and in particular to the superpotential of [10]. We believe that the expansion analysis of [10], based on integrating out $S$ and analyzing the spectrum of light states in terms of coefficients of dimension 6 operators, must be breaking down, and this explains the discrepancy.

We conclude this Section by analyzing the effect of extra $S U(5)$ multiplets on the gauge coupling unification. In Table 1 we show the prediction of $\alpha_{S}\left(M_{Z}\right)$ for $n_{5}=3$ from the running of the gauge couplings at one and two loops, compared with the standard case $\left(n_{5}=0\right)$, without any threshold effect. In the same Table we give, for the two cases, the corresponding value of the unified coupling $\alpha_{G}$. As is well known, the one loop prediction is very close to the experimental value $\alpha_{S}\left(M_{Z}\right)=0.1176(20)$, and of course this conclusion is left unchanged by the addition of extra matter in full $S U(5)$ multiplets. At two loops, the prediction for $n_{5}=3$ is brought closer to the experiment compared to the standard $n_{5}=0$ result. However, the unavoidable presence of threshold corrections does not allow a significant distinction between the two cases. In fact, a $i$-th $(5+\overline{5})$ split into a $S U(3)$-triplet of mass $M_{d i}$ and a $S U(2)$ doublet of mass $M_{L i}, \alpha_{S}\left(M_{Z}\right)$ gives a further one loop threshold correction

$$
\frac{\delta \alpha_{S}\left(M_{Z}\right)}{\alpha_{S}\left(M_{Z}\right)} \simeq \frac{9 \alpha_{S}\left(M_{Z}\right)}{14 \pi} \log \frac{M_{d i}}{M_{L i}} \approx 2 \% \log \frac{M_{d i}}{M_{L i}}
$$

There is furthermore a two loop contribution from $\lambda$ itself, dominated by the UV,

$$
\frac{\delta \alpha_{S}\left(M_{Z}\right)}{\alpha_{S}\left(M_{Z}\right)} \simeq-\frac{9 \alpha_{S}\left(M_{Z}\right)}{56 \pi} \log \left(\frac{\lambda_{G U T}^{2}}{2 \pi^{2}} \log \frac{M_{G U T}}{M_{Z}}\right)
$$

i.e., numerically, $\delta \alpha_{S}\left(M_{Z}\right) / \alpha_{S}\left(M_{Z}\right) \simeq-(1 \div 2) \%$ for $\lambda_{\mathrm{GUT}} / 4 \pi=0.3 \div 0.2$. 

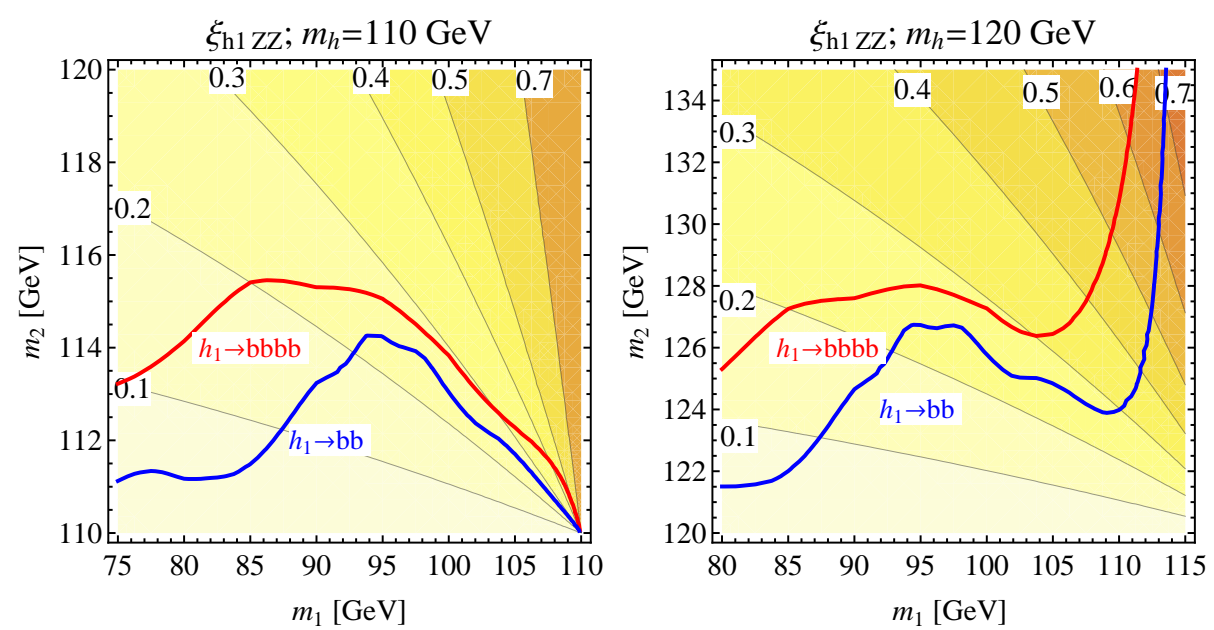

Figure 3: For two reference values $m_{h}=110 \mathrm{GeV}$ (left) and $m_{h}=120 \mathrm{GeV}$ (right) we plot the normalized squared coupling, eq. (3.2), of the lightest scalar $h_{1}$ in the $2 \times 2$ mixing model eq. (3.1), plotted as a function of its mass $m_{1}$ and the mass of the heavier state $m_{2}$. The region below the lower blue (upper red) curve is consistent with the $95 \%$ C.L. bounds [16] from nonobservation of $h_{1}$ at LEP2, assuming it decays into $b \bar{b}(b \bar{b} b \bar{b})$. The heavier scalar $h_{2}$, to be consistent with the LEP2 searches, should have the mass above $\sim 114 \mathrm{GeV}$.

\section{A simple $2 \times 2$ mixing model}

As already mentioned, what really matters for the NMSSM phenomenology, more than $m_{h}$ itself, are the masses and compositions of the physical scalars. Before mixing with $h$, the MSSM has two other CP-even fields, $s_{1}, s_{2}$ (in their mass-squared diagonal basis). Both their masses and compositions depend on all the various parameters of the NMSSM. Nevertheless, none of them is coupled to VV.

Mixing of $h$ with $s_{1}$ and $s_{2}$ (if one, or perhaps both, of these states are lighter than $h$ ) can help increase the mass of $h$. After the mixing, $s_{1}$ and $s_{2}$ acquire coupling to VV and become subject to LEP searches. As we are going to see, these mixing cannot be large for consistency with LEP. As such, one can analyze individually their additive effects without making any significant error. We can then consider a simplified $2 \times 2$ mixing mode $5^{5}$ between $h$ and the lightest, $s_{1}$, among the two states not coupled to ZZ. Thus we consider a mass matrix

$$
\mathcal{M}_{2 \times 2}^{2}=\left(\begin{array}{cc}
m_{h}^{2} & \Delta m^{2} \\
\Delta m^{2} & m_{s_{1}}^{2}
\end{array}\right)
$$

with a fixed $m_{h}$ and arbitrary $m_{s_{1}}<m_{h}$ and $\Delta m^{2}$.

In view of the previous Section and having in mind the LEP bound of about $115 \mathrm{GeV}$, only valid for the SM Higgs boson, we take for $m_{h}$ two reference values, 110 and $120 \mathrm{GeV}$, close to

\footnotetext{
${ }^{4}$ In fact they used the soft stop mass of $300 \mathrm{GeV}$, which corresponds to physical stop mass of $345 \mathrm{GeV}$, an irrelevant difference.

${ }^{5}$ The effects of a $2 \times 2$ mixing model of this type has already been considered in the NMSSM $[9]$, $[5$ and in the MSSM as well [12, [13, ,14,,15].
} 
the upper bounds on $m_{h}$ without or with extra matter respectively. In absence of mixing only the latter case would be compatible with LEP data. With mixing, however, which is generally present, the situation may change.

In Fig.s 3, we describe the effect of mixing $h$ with $s_{1}$ in the two cases. In the plane of the two mass eigenvalues $\left(m_{1}, m_{2}\right)$ - from which we can uniquely reconstruct $m_{s_{1}}^{2}$ and $\Delta m^{2}$ - we give the isolines of the squared coupling of the lightest state to ZZ, normalized to the SM Higgs boson coupling:

$$
\xi_{h_{1} Z Z}=\left(\frac{g_{h_{1} Z Z}}{g_{h Z Z}}\right)^{2} .
$$

From the data of Ref. [16] this allows to determine in the same plane the 95\% C.L. bound from the non-observation of the lightest state, assumed to decay in $b \bar{b}$ with SM branching ratio. For later purposes we also consider the decay in $b \bar{b} b \bar{b}$ with a branching ratio close to 1 . Given the actual numbers, a quick way to understand from these figures the compatibility with LEP data is to see if there are values of the heaviest mass $m_{2}$ above $115 \mathrm{GeV}$ and simultaneously allowed by the bound on the lightest state.

The conclusions are quite clear. With an unmixed value of $m_{h}=110 \mathrm{GeV}$, and a fortiori for lower values, it is hardly possible to obtain consistency with the LEP data 6 . This means that, with a moderate stop mass and a small $A_{t}$-term, the NMSSM without extra matter and with standard Higgs boson decays can perhaps be accommodated with LEP data, if at all, only in a small corner of its parameter space. This may explain the interest of considering the decay of the lightest state into $\tau \bar{\tau} \tau \bar{\tau}$, which is experimentally less constrained [3].

On the other hand, the $m_{h}=120 \mathrm{GeV}$ case is obviously compatible with LEP data for small enough mixing. More important is that some mixing effects will inevitably be present, which can push the heavier state even further up with a somewhat reduced coupling to the ZZ, while keeping consistency with the LEP data for the lower state. This can be a characteristic feature of the NMSSM with extra matter contributing to the RGE running of the coupling constants, and is the phenomenological pattern to which we want to draw attention.

\section{An explicit example based on an approximate Peccei- Quinn Symmetry: PQ SUSY}

\subsection{The Lagrangian and the allowed parameter space}

An independent motivation for the NMSSM is that it may provide a simple solution of the so called $\mu$-problem: the supersymmetric superpotential mass term $\mu H_{1} H_{2}$ gets replaced by $\lambda\langle S\rangle H_{1} H_{2}$ and all the mass terms in the Lagrangian originate from supersymmetry breaking. This possible solution of the $\mu$-problem invites a symmetry explanation of the absence of mass terms in the superpotential. Such symmetries can be a continuous R-invariance and/or a Peccei-Quinn (PQ) symmetry. In this paper we choose a PQ symmetry since: i) it removes the $\frac{\kappa}{3} S^{3}$ coupling, thereby

\footnotetext{
${ }^{6}$ However, notice the point at $\left(m_{1}, m_{2}\right) \approx(95,115) \mathrm{GeV}$ which has been emphasized in the literature 13 in connection with a slight excess of events at LEP.
} 
helping to maximize $\lambda$ at the weak scale; ii) it can reduce the number of parameters in the supersymmetry breaking Lagrangian as well, since PQ may be approximately realized in this sector without conflicting with experiments. This version of NMSSM, which we call "PQ SUSY," has a minimal number of parameters, and contains a light pseudo-Goldstone boson. For earlier considerations of NMSSM in the PQ limit, see [6], [7].

Up to the small breaking of the PQ symmetry, the Lagrangian is uniquely fixed by the superpotential term

$$
f=\lambda S H_{1} H_{2},
$$

by the soft non-supersymmetric piece of the scalar potential

$$
V_{\text {soft }}=m_{S}^{2}|S|^{2}+m_{1}^{2}\left|H_{1}\right|^{2}+m_{2}^{2}\left|H_{2}\right|^{2}+\left(A_{\lambda} \lambda S H_{1} H_{2}+\text { H.c. }\right)
$$

and by the gaugino mass terms, which we shall take large relative to $\lambda\langle S\rangle$ (see below). Small breaking terms of the PQ symmetry, like $\delta V=m^{2} S^{2}+B \mu H_{1} H_{2}+$ H.c., will have to be present. However we assume them to be small enough only to give mass to the otherwise massless pseudoGoldstone boson, without significantly affecting any of the remaining properties of the model. We have checked that this is a consistent approximation.

When it exists, the CP-conserving, $S U(2) \times U(1) \rightarrow U(1)$ breaking vacuum is related to the Lagrangian parameters by $\left(x=m_{S}^{2} / \lambda^{2} v^{2}\right)$

$$
\begin{gathered}
\lambda^{2} v^{2}=M_{Z}^{2}+\frac{A_{\lambda}^{2}}{1+x}+\frac{m_{1}^{2}-m_{2}^{2}}{\cos 2 \beta}, \\
\sin ^{2} 2 \beta=2\left[(1+x)-(1+x)^{2} \frac{m_{1}^{2}+m_{2}^{2}+\lambda^{2} v^{2}}{A_{\lambda}^{2}}\right], \\
\langle S\rangle \equiv v_{s}=\frac{A_{\lambda}}{2 \lambda(1+x)} \sin 2 \beta .
\end{gathered}
$$

Note that the scalar sector defined by eq.s (4.1 4.2 depends upon five parameters (apart from the pseudo-Goldtone mass $\left.m_{G}\right): \lambda$ and $m_{1}^{2}, m_{2}^{2}, m_{S}^{2}, A_{\lambda}$. We trade $m_{1,2}^{2}$ for $v$ and $\tan \beta$. A useful way to represent the various results, which we shall follow, is to show them in the plane $\left(m_{S}, A_{\lambda}\right)$ for fixed values of $\lambda, \tan \beta$. In particular the vacuum in eq.s 4.3, 4.4, 4.5) is indeed the true minimum of the overall potential only in a portion of this parameter space. Fig. 4 shows the allowed parameter space for $\tan \beta=1.5,2,2.5$ and $\lambda$ close to the maximal allowed values from Fig. 1. We see that $A_{\lambda}$ has a maximal and minimal allowed value for each $m_{S}$ in an interval $0<m_{S}<m_{S}^{\max } \simeq 70 \mathrm{GeV}$. Here $m_{S}^{2}>0$ is required by the global stability. The upper limit on $A_{\lambda}$ comes from imposing that $V<0$ at the minimum $4.3,4.4,4.5$, so that it is preferred to the trivial stationary point at $v_{1}=v_{2}=v_{s}=0$. The lower limit on $A_{\lambda}$ comes from the experimental bound on the chargino mass, $m\left(\chi^{ \pm}\right)>103 \mathrm{GeV}$ [17], via Eq. (4.14) below. The constraint of local stability does not further restrict the parameter space. 


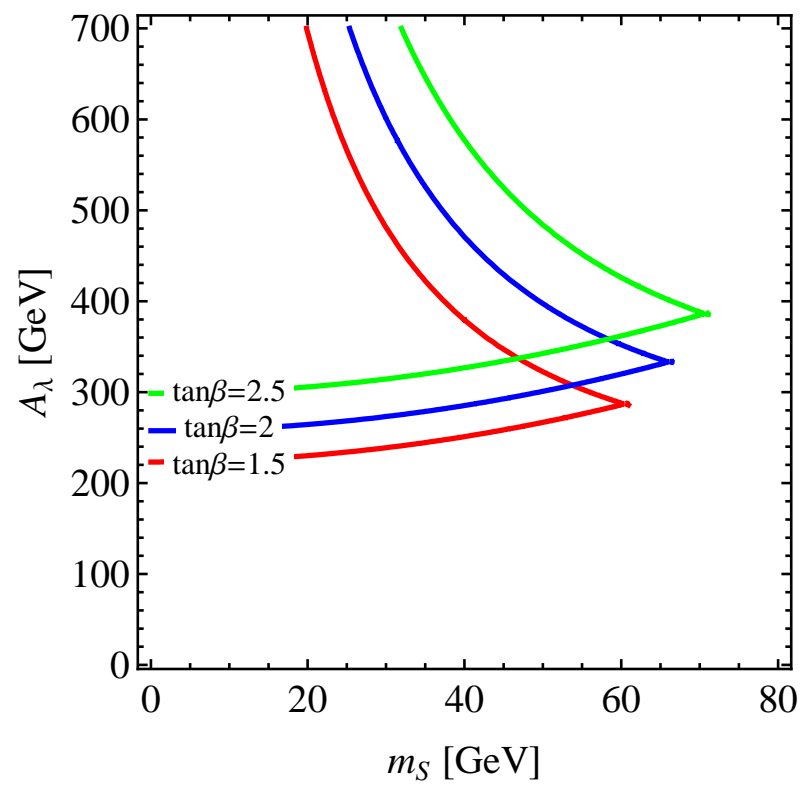

Figure 4: The allowed region of the $\left(m_{S}, A_{\lambda}\right)$ plane (see the text) for $\tan \beta=1.5,2,2.5$ and $\lambda$ fixed at $0.65,0.7,0.75$, respectively.

\subsection{Higgs boson and higgsino spectra}

The spectrum of the Higgs boson sector is straightforwardly obtained by expanding around the above minimum. For the single charged boson one finds

$$
m_{H^{ \pm}}^{2}=M_{W}^{2}+\frac{A_{\lambda}^{2}}{1+x}-\lambda^{2} v^{2} .
$$

Out of the two neutral CP-odd states, one is massless in this approximation (the PQ Goldstone $G)$ and the other has mass

$$
m_{A}^{2}=\frac{A_{\lambda}^{2}}{1+x}+\lambda^{2} v^{2}(1+x) .
$$

Their compositions in terms of the neutral fields

$$
H_{1}^{0}=\frac{1}{\sqrt{2}}\left(h_{1}+i \pi_{1}\right), H_{2}^{0}=\frac{1}{\sqrt{2}}\left(h_{2}+i \pi_{2}\right), S=\frac{1}{\sqrt{2}}\left(s+i \pi_{s}\right),
$$

are

$$
\begin{aligned}
G & =-\sin \alpha \pi_{s}+\cos \alpha\left(\cos \beta \pi_{2}-\sin \beta \pi_{1}\right), \\
A & =\cos \alpha \pi_{s}+\sin \alpha\left(\cos \beta \pi_{2}-\sin \beta \pi_{1}\right),
\end{aligned}
$$

where

$$
\tan \alpha=\frac{A_{\lambda}}{\lambda v(1+x)} .
$$

The $3 \times 3$ squared mass matrix of the CP-even neutral scalars is best written in the basis

$$
\left(H=\cos \beta h_{2}-\sin \beta h_{1}, h=\cos \beta h_{1}+\sin \beta h_{2}, s\right)
$$




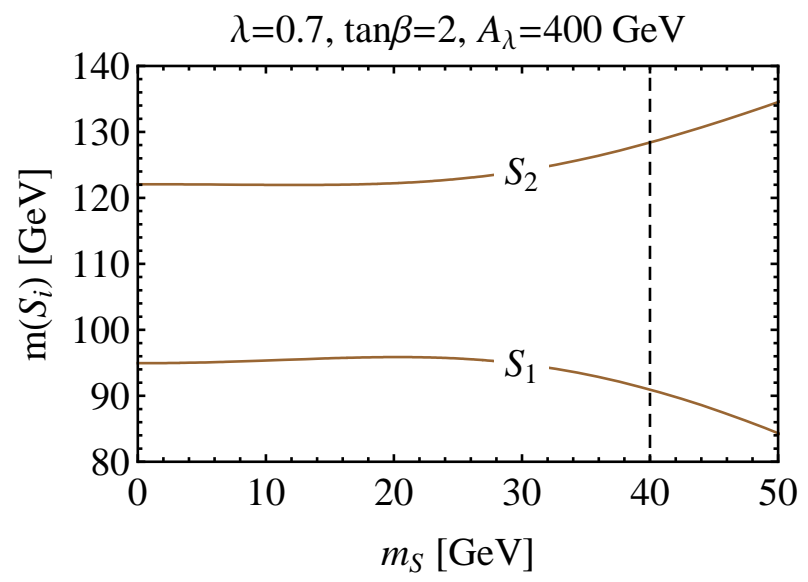

Figure 5: The masses of the two lightest CP-even scalars in the model of Section 4 for $\left(\lambda, \tan \beta, A_{\lambda}\right)=(0.7,2,400 \mathrm{GeV})$ and a range of $m_{S}$ values consistent with global stability of the scalar potential. We include the stop quantum correction with $m_{\tilde{t}}=300 \mathrm{GeV}$. The region to the right of the dashed line $\left(m_{S}>40 \mathrm{GeV}\right)$ is excluded by LEP constraints on the $S_{1} Z Z$ coupling (see Fig. 6).

where it has the form

$$
\mathcal{M}^{2}=\left(\begin{array}{ccc}
\frac{A_{\lambda}^{2}}{1+x}+\left(M_{Z}^{2}-\lambda^{2} v^{2}\right) \sin ^{2} 2 \beta & -\frac{1}{2}\left(M_{Z}^{2}-\lambda^{2} v^{2}\right) \sin 4 \beta & -A_{\lambda} \lambda v \cos 2 \beta \\
* & M_{Z}^{2} \cos ^{2} 2 \beta+\lambda^{2} v^{2} \sin ^{2} 2 \beta & -A_{\lambda} \lambda v \sin 2 \beta \frac{x}{1+x} \\
* & * & \lambda^{2} v^{2}(1+x)
\end{array}\right) .
$$

Note, as anticipated, that $\mathcal{M}_{22}^{2}=\left(m_{h}^{0}\right)^{2}$. Note also that one of the mixing terms between $h$ and the two other scalars, $\mathcal{M}_{12}^{2}$ is always small, whereas the other, $\mathcal{M}_{23}^{2}$, is essentially controlled by $x$ (or $m_{S}^{2}$ ). The mixing pattern discussed in the previous Section is precisely realized in this case, as shown in Fig. 5, where one has the two lightest scalar masses as functions of $m_{S}$ for $\left(\lambda, \tan \beta, A_{\lambda}\right)=(0.7,2,400 \mathrm{GeV})$. The lightest scalar mass is below the LEP limit; its dominant decay mode (see the next Section) is into 2 PQ pseudo-Goldstones: $S_{1} \rightarrow G G \rightarrow 4 b$. From Fig. 6 we see that for $(\lambda, \tan \beta)=(0.7,2)$ the LEP constraint on the $S_{1}$ coupling to $\mathrm{ZZ}$ is satisfied in most of the parameter space allowed by the potential stability and the chargino mass bound 7 . The heaviest CP-even scalar has mass $m_{S_{3}} \approx A_{\lambda} /(1+x)^{1 / 2}$ which for $A_{\lambda}=400 \mathrm{GeV}$ is in the $380 \div 400 \mathrm{GeV}$ range, while $A$ and $H^{ \pm}$are $\sim 15 \mathrm{GeV}$ heavier and lighter than $S_{3}$, respectively.

For heavy gaugino masses, the masses of the higgsinos are controlled by the effective $\mu$ parameter

$$
\mu=\lambda v_{s}=\frac{A_{\lambda}}{2(1+x)} \sin 2 \beta .
$$

The single charged higgsino has mass $m\left(\chi^{ \pm}\right)=\mu$, whereas the $3 \times 3$ neutralino mass matrix in

\footnotetext{
${ }^{7}$ The processes $e^{+} e^{-} \rightarrow Z^{*} \rightarrow S_{1,2} G$ followed by $S_{1,2} \rightarrow G G$ could not possibly be seen at LEP2: the normalized squared couplings $\xi_{Z S_{1,2} G}$ are tiny, $\lesssim 10^{-2}$, one order of magnitude below the LEP2 limits [16].
} 


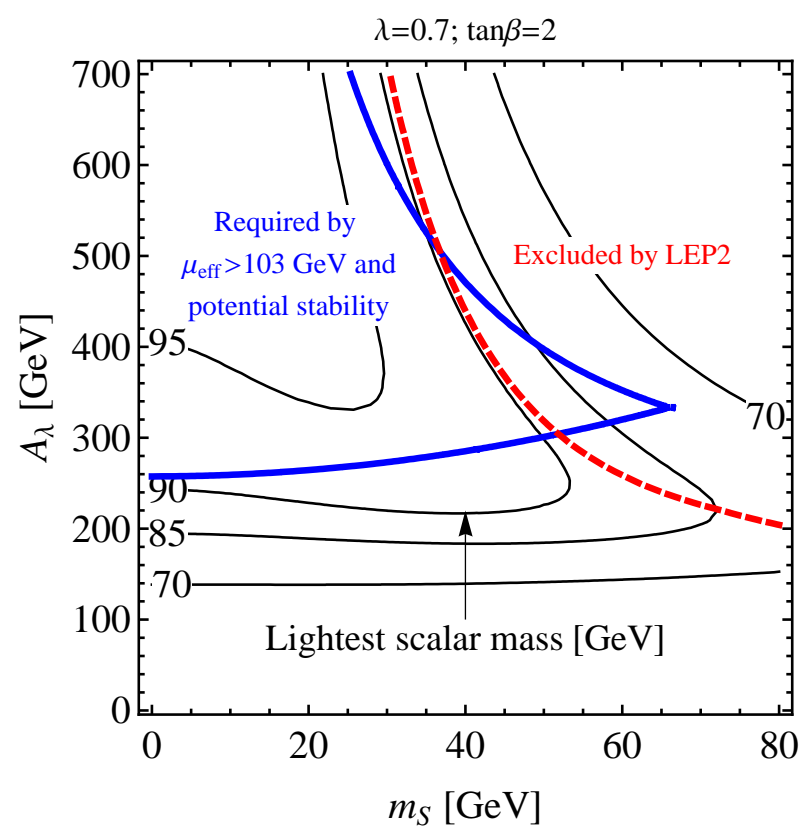

Figure 6: For $(\lambda, \tan \beta)=(0.7,2)$ we give the allowed regions in $\left(m_{S}, A_{\lambda}\right)$ plane following from the stability of the potential and the chargino mass bound (inside the blue curve, same as in Fig. (4), and from the non-observation of $S_{1} \rightarrow G G \rightarrow 4 b$ decays at LEP2 (to the left of the dashed red curve). In the same plot we show contours of the lightest CP-even scalar mass. As always, we include the stop quantum correction with $m_{\tilde{t}}=300 \mathrm{GeV}$. 


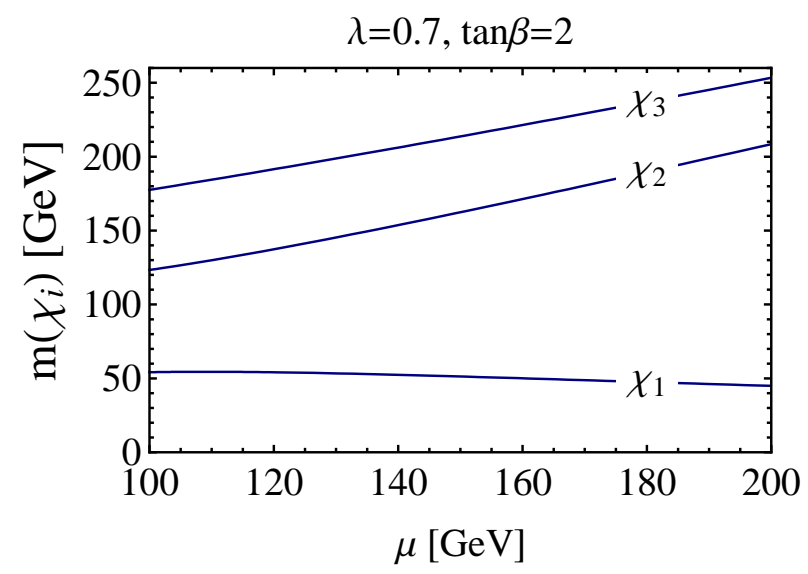

Figure 7: The neutralino masses in the model of Section 4 for $(\lambda, \tan \beta)=(0.7,2)$ and the chargino mass $\mu$ in its typical range. We have $m\left(\chi_{1}\right)+m\left(\chi_{2}\right)=m\left(\chi_{3}\right)$ since the neutralino mass matrix 4.15 is traceless.

the basis $\left(\tilde{h}_{1}, \tilde{h}_{2}, \tilde{s}\right)$ has the form

$$
\mathcal{M}_{\chi}=\left(\begin{array}{ccc}
0 & \mu & \lambda v \sin \beta \\
* & 0 & \lambda v \cos \beta \\
* & * & 0
\end{array}\right) .
$$

Fig. 7 shows the values of the neutralino masses versus $m\left(\chi^{ \pm}\right)$in its typical range, $100 \div 200 \mathrm{GeV}$, for $(\lambda, \tan \beta)=(0.7,2)$. For these masses the LEP2 searches have not been possibly effective. Indeed, the process $e^{+} e^{-} \rightarrow Z^{*} \rightarrow \chi_{1} \chi_{2}$ is within the LEP2 kinematic limit in a part of the parameter space (see Fig. 7). However, the production cross section turns out to be well below the $\sim 0.1 \mathrm{pb}$ limit set in [17] due to phase space and coupling suppressions.

Another possible process is $e^{+} e^{-} \rightarrow \chi_{1} \chi_{1} \gamma_{\text {ISR }}$ with a photon (from Initial State Radiation) and missing transverse energy in the final state, which is constrained by LEP2 searches of extra neutrino species [18]. However, we concluded that the existing data cannot rule out a $\chi_{1}$ with a somewhat reduced $Z \chi_{1} \chi_{1}$ coupling and mass above $m_{Z} / 2$, as it is in our case (see also [19]).

If the gravitino is the lightest SUSY particle, the lightest neutralino will predominantly decay into the gravitino and the pseudo Goldstone boson $G$. However, if the SUSY breaking scale $\sqrt{F}$ exceeds about $1000 \mathrm{TeV}$, these decays happen outside the detector and do not modify collider phenomenology of the model (see Section 5).

\subsection{Higgs boson couplings and branching ratios}

The phenomenology of the model is made peculiar by the presence of the light pseudoscalar $G$, with an unknown mass, $m_{G}$, coming from the breaking of the PQ symmetry and assumed to be relatively small.

If $m_{G}$ is below the $b \bar{b}$ threshold, the pseudoscalar can be seen in radiative $\Upsilon$ decays [20]. The 


\begin{tabular}{|l|l|l|}
\hline & Production coupling & Branching ratios \\
\hline$S_{1}$ & $\xi_{S_{1} t t}, \xi_{S_{1} V V} \lesssim 20 \%$ (Fig. 8) & $B R(G G) \geq 98 \%)$ \\
\hline \multirow{3}{*}{$S_{2}$} & $\xi_{S_{2} t t}, \xi_{S_{2} V V} \simeq 100 \%$ & See Fig.9: \\
& & $B R\left(\chi_{1} \chi_{1}\right)=50 \div 90 \%$ \\
& & $B R(G G) \simeq 1-B R\left(\chi_{1} \chi_{1}\right)$ \\
\hline \multirow{3}{*}{$S_{3}$} & $\xi_{S_{3} t t} \simeq 20 \%, \xi_{S_{3} V V}$ negligible & See Fig.9: \\
& & $B R\left(\chi_{i} \chi_{j}\right) \simeq 35 \%\left(\right.$ of which $50 \%$ into $\left.\chi_{1} \chi_{1}\right)$ \\
& & $B R(Z G) \simeq 30 \%$ \\
& $B R\left(S_{i} S_{j}\right) \simeq 20 \%$ \\
\hline
\end{tabular}

Table 2: The neutral CP-even Higgs boson dominant decay modes and the couplings relevant for their production at the LHC via gluon fusion and vector boson fusion processes, for $\left(\lambda, \tan \beta, A_{\lambda}\right)=(0.7,2,400 \mathrm{GeV})$ and $0<m_{S} \lesssim 40 \mathrm{GeV}$.

relevant branching ratio is given by [21]

$$
\begin{aligned}
B R(\Upsilon \rightarrow \gamma G) & =F(\cos \alpha)^{2}(\tan \beta)^{2} \frac{G_{F} m_{b}^{2}}{\sqrt{2} \pi \alpha_{\mathrm{EM}}} B R\left(\Upsilon \rightarrow \mu^{+} \mu^{-}\right) \\
& \simeq 2 \times 10^{-4} F(\cos \alpha)^{2}(\tan \beta)^{2}, \quad F=\left(1-\frac{m_{G}^{2}}{m_{\Upsilon}^{2}}\right) F_{0},
\end{aligned}
$$

where $\alpha$ is the angle in (4.11), and the suppression factor $F_{0} \lesssim 0.5$ is due to QCD, bound state and relativistic corrections (see [22], Section 3.1). For $\left(\lambda, \tan \beta, A_{\lambda}\right)=(0.7,2,400 \mathrm{GeV})$ we get $B R(\Upsilon \rightarrow \gamma G) \simeq 0.5 \times 10^{-5}$. The experimental limits on this branching ratio depends crucially on the decay properties of $G$. An interesting possibility occurs if $2 m_{\tau}<m_{G}<2 m_{b}$, so that $G$ decays into $\tau^{+} \tau^{-}$. In this case the current limit from CLEO is [23]

$$
B R(\Upsilon \rightarrow \gamma G(\rightarrow \tau \tau)) \lesssim 10^{-4}
$$

and a dedicated run by BABAR may improve it soon by $1-2$ orders of magnitude.

Below we will assume that $m_{G}$ is above the $b \bar{b}$ threshold, corresponding to a relatively less restricted region of parameter space. The pseudoscalar then decays into $b \bar{b}$ and $\tau \bar{\tau}$ with branching ratios close to the branching ratios of the SM Higgs boson.

All the couplings and decay rates for the other Higgs bosons are easily determined from the parameters of the model as given in the previous Section. Table 2 and Figs. 89 illustrate the main features of the most relevant quantities for $\left(\lambda, \tan \beta, A_{\lambda}\right)=(0.7,2,400 \mathrm{GeV})$ and $0<m_{S} \lesssim 40$ GeV (see Fig. 6).

Using these numbers one can make a preliminary conclusion that observing these states at the LHC will not be easy, since the production cross sections are suppressed, and the dominant decay products do not allow for easy background discrimination. Obviously, a more detailed study is required to assess the LHC discovery potential. 


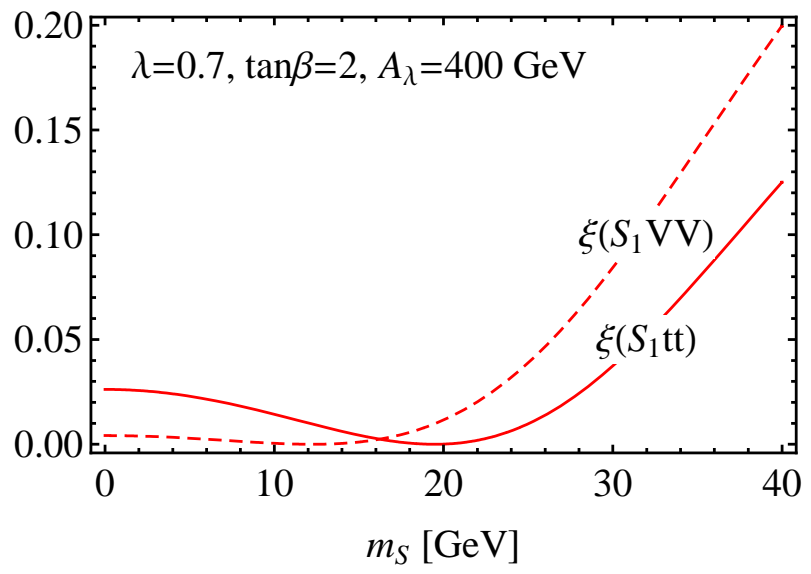

Figure 8: The $S_{1} t \bar{t}$ and $S_{1} V_{\mu} V^{\mu}$ couplings squared of the lightest CP-even scalar $S_{1}$, normalized to the couplings of the SM Higgs boson analogously to Eq. (3.2). In this plot $\left(\lambda, \tan \beta, A_{\lambda}\right)=(0.7,2,400 \mathrm{GeV})$.
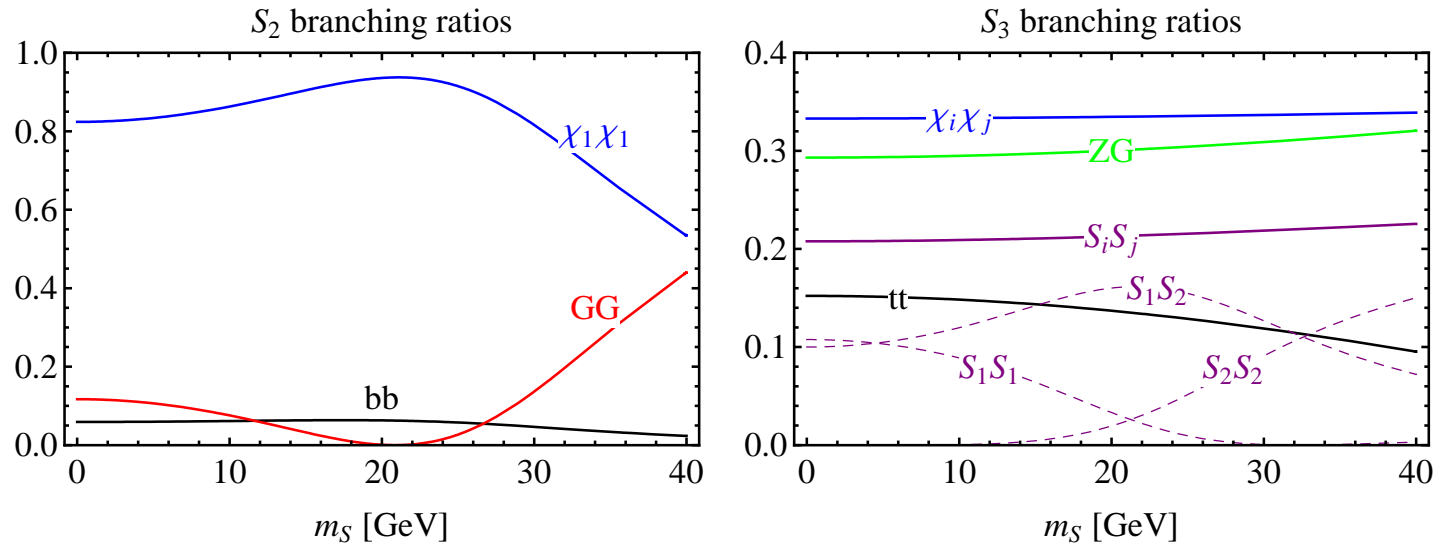

Figure 9: The dominant branching ratios of $S_{2}$ (left) and $S_{3}$ (right) for $\left(\lambda, \tan \beta, A_{\lambda}\right)=$ $(0.7,2,400 \mathrm{GeV})$. 


\subsection{Fine tuning}

It is interesting to know an estimate of the finetuning required to satisfy the various restrictions on the parameters of the model under consideration. For an early discussion of finetuning in the NMSSM see [24]. The first thing to check is if there is too strong dependence of the Z-mass, or of the vacuum expectation value $v$ in eq. (4.3), on the various parameters. The strongest such dependence is on the parameter $A_{\lambda}^{2}$. Naively this would seem to require finetuning of the order $A_{\lambda} / \lambda^{2} v^{2} \sim 10$ for $A_{\lambda}=400 \mathrm{GeV}$. However, this estimate does not take into account the fact that the two cancelling terms are not totally independent: the variation of $A_{\lambda}$ influences the other term via the angle $\beta$ as determined by the second equation (4.4). An estimate which takes this effect into account is given by the logarithmic derivative ${ }^{8}$

$$
\Delta=\left|\frac{\partial \log v^{2}}{\partial \log A^{2}}\right|,
$$

which can be evaluated numerically, see Fig. 10 . We see that $\tan \beta$ below $\sim 1.7$ starts to be disfavored by this finetuning, although $\tan \beta=2, A_{\lambda}=400 \mathrm{GeV}$ is OK with less than $10 \%$ finetune.

Whereas these considerations apply to the dependence of $v$ on the low energy parameters, it is also necessary to check the consistency of the values of these same parameters with the expected contributions due to RGE evolution? Here the difference $m_{2}^{2}-m_{1}^{2}$ gets a contribution due to the stop mass:

$$
\delta\left(m_{2}^{2}-m_{1}^{2}\right)=\frac{3 y_{t}^{2}}{4 \pi^{2}}\left(m_{\tilde{t}}^{2}-m_{t}^{2}\right) \log \frac{\Lambda_{\text {mess }}}{v} \text { (no mixing) }
$$

where $\log \Lambda_{\text {mess }} / v=6 \div 40$ for $\Lambda_{\text {mess }}=100 \mathrm{TeV} \div 10^{19} \mathrm{GeV}$. This equation can be used to set an upper bound on the stop mass with no finetuning, i.e. for $\delta\left(m_{2}^{2}-m_{1}^{2}\right) /\left(m_{2}^{2}-m_{1}^{2}\right)=1$, see Fig 11 , where we take low mediation scale, $\Lambda_{\text {mess }}=100 \mathrm{TeV}$. We see that $m_{\tilde{t}}=300 \mathrm{GeV}$, as assumed above, is safely within the allowed range.

Finally we notice that, although $v$ in eq. (4.3) is only weakly dependent on $m_{S}^{2}$, the current experimental constraints mostly on the chargino mass (see eq. (4.14) and Fig. 4) require $m_{S}$ to be below $40 \div 50 \mathrm{GeV}$. At the same time there is a one-loop contribution to the running of $m_{S}^{2}$ due to $A_{\lambda}^{2}$ :

$$
\delta m_{S}^{2}=\frac{1}{4 \pi^{2}} \lambda^{2} A_{\lambda}^{2} \log \frac{\Lambda_{\text {mess }}}{v}
$$

For $\lambda=0.7, A_{\lambda}=400 \mathrm{GeV}$, and $\Lambda=100 \mathrm{TeV}$ this gives $\delta m_{S}^{2}=(110 \mathrm{GeV})^{2}$. Since the allowed range of $m_{S}^{2}$ is a factor $5 \div 10$ smaller, to comply with this limit it is clear that the model under consideration would again prefer low mediation scale.

\footnotetext{
${ }^{8} \mathrm{As}$ a consequence of the above effect the derivative $\partial v^{2} / \partial A^{2}$ is actually negative.

${ }^{9}$ Alternatively we could look directly at the dependence of $v$ on the high energy paremeters, which are considered more fundamental.

${ }^{10}$ To make this plot, parameters $m_{1}^{2}$ and $m_{2}^{2}$ have to be expressed in terms of $v, \lambda, \tan \beta, A_{\lambda}$ and $m_{S}$ from equations 4.3 , 4.4 .
} 


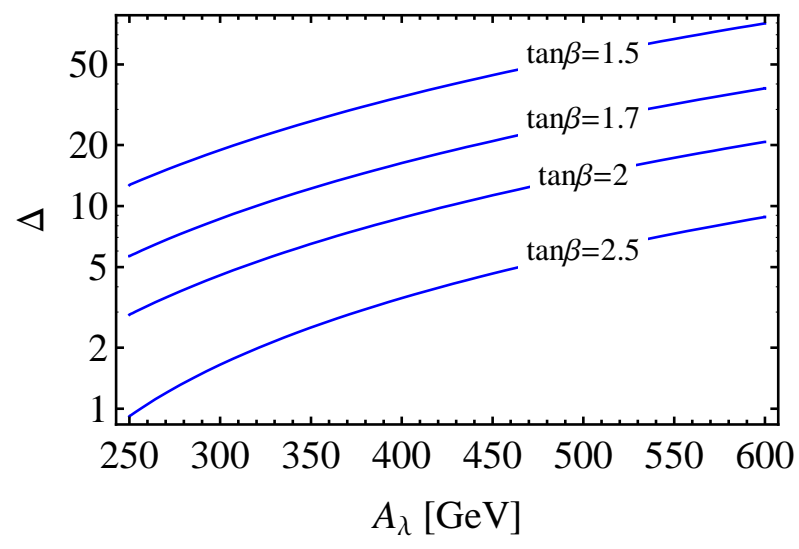

Figure 10: The logarithmic derivative Eq. 4.16 plotted as a function of $A_{\lambda}$ for $(\tan \beta, \lambda)=$ $(1.5,0.65),(1.7,0.7),(2,0.7),(2.5,0.75)$. Smaller $\tan \beta$ give bigger $\Delta$ (and hence require bigger finetuning). In this plot we assumed $m_{S}=0$, but the change for small allowed values of $m_{S}$ is negligible.

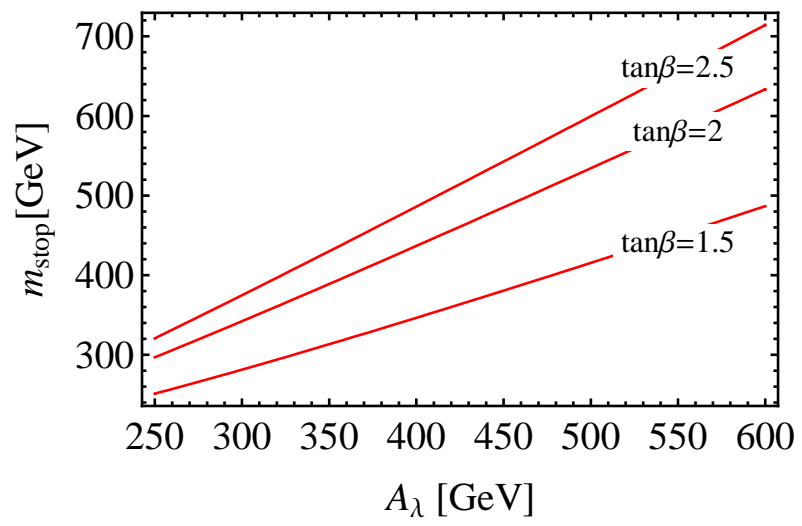

Figure 11: The maximal value of physical stop mass (with no mixing) which is consistent with the naturalness bound $\delta\left(m_{2}^{2}-m_{1}^{2}\right) /\left(m_{2}^{2}-m_{1}^{2}\right)<1$, see Eq. 4.17). From below up: $(\tan \beta, \lambda)=(1.5,0.65),(2,0.7),(2.5,0.75)$. Mediation scale $\Lambda_{\text {mess }}=100 \mathrm{TeV}$. In this plot we assumed $m_{S}=0$, but the change for small allowed values of $m_{S}$ is negligible. 


\section{Conclusions and outlook}

Even if we assume, with good reasons indeed, that supersymmetry is relevant in nature, there is no water-tight argument that requires the presence of supersymmetric signals at the LHC. Our best hope is a natural solution of the hierarchy problem of the Fermi scale, which makes such a presence likely. For this to be the case, however, requires a low level of fine tuning in the $Z$-mass, i.e. a maximally natural solution of the hierarchy problem. The LEP limit on the Higgs boson mass is particularly important, since it excludes the most natural regions of parameter space of the simplest supersymmetric models. This amply motivates the focus on supersymmetric extensions of the SM that minimize this fine-tuning and remain, at the same time, reasonably simple.

A particularly simple possibility for increasing the Higgs mass is to add a new quartic interaction for the Higgs doublets via the superpotential interaction $\lambda S H_{1} H_{2}$. However, to maintain perturbative unification of the gauge couplings, a clear success of weak scale supersymmetry, the value of $\lambda$ is limited. With minimal matter content this interaction provides at most an additional $\sim 10 \mathrm{GeV}$ to the Higgs boson mass, leading to only a small allowed region of parameter space, even including mixing amongst the Higgs bosons. In contrast, with additional matter the perturbative evolution of couplings allows a larger value of $\lambda$, increasing the Higgs boson mass by up to $\sim 20 \mathrm{GeV}$ compared to the theory without the singlet field. Furthermore, in this case mixing can augment the Higgs boson mass by another $2-8 \mathrm{GeV}$, considerably enlarging the allowed region of parameter space.

The extra matter implies that the gauge couplings are larger in the UV, and the top coupling smaller, compared to the minimal matter case. Providing the extra matter fills complete SU(5) multiplets, the successful unification of gauge couplings at 1 loop is unaltered. The changes from 2 loops and threshold corrections depends on the nature of the extra matter. In the case of $5+\overline{5}$ representations, the prediction for $\alpha_{s}\left(M_{Z}\right)$ from 2 loop running is decreased, improving the agreement with data, but this is offset by an expected increase in $\alpha_{s}\left(M_{Z}\right)$ from threshold corrections from non-degeneracies within the $5+\overline{5}$ multiplets. The situation with $10+\overline{10}$ representations is the opposite: 2 loop running increases the discrepancy with data, but is countered by the threshold corrections. In either case, the significance of gauge coupling unification is comparable to the standard case with minimal matter.

In this work we have considered PQ SUSY - a version of the NMSSM that incorporates the above mechanism for enlarging the Higgs mass and is fully realistic, with a minimum number of parameters. The superpotential is assumed to be exactly invariant under a Peccei-Quinn symmetry, elegantly solving the $\mu$ problem, while the soft scalar interactions include small PQ breaking interactions to give a mass to the pseudo-Goldstone boson $G$. The theory possesses just two parameters more than the MSSM - one is the soft mass parameter for the scalar $S, m_{S}^{2}$, and the other is the mass for $G$. A combination of vacuum stability and chargino mass limits implies a restricted range for $m_{S}^{2}$, so that a $10-20 \%$ fine tuning is necessary, and a low messenger scale is preferred.

The Higgs boson system has a few characteristic properties in its spectrum and in its couplings. The spectrum contains two CP-even neutral scalars relatively close in mass, one above and one below the "naive" LEP bound of $115 \mathrm{GeV}$ by $10 \div 20 \mathrm{GeV}$, and with a shared coupling to the vector boson pairs, VV. Only the sum of these coupling squared is close to the squared coupling of 
the SM Higgs boson to VV. Related to the approximate Peccei-Quinn symmetry, the Higgs boson spectrum also contains a CP-odd light state, $G$, present in the main decay modes of all the CP even neutral scalars. $G$ itself decays to $b \bar{b}$ and $\tau \bar{\tau}$, with branching ratios close to those of a light SM Higgs boson. Quite clearly, to assess the discovery potential of such a Higgs boson system at the LHC or TeVatron requires, and deserves, a detailed examination.

A small region of parameter space where $2 m_{\tau}<m_{G}<2 m_{b}$ and $G$ decays into $\tau^{+} \tau^{-}$is also allowed; in this case the model predicts $B R(\Upsilon \rightarrow \gamma G)$ within reach of the existing B-factories.

The general phenomenology of the model crucially depends on the properties of the lightest neutralino, which is predicted to have a mass near $50 \mathrm{GeV}$. Naturalness considerations suggest a low scale for supersymmetry breaking, $\sqrt{F}$, so that the gravitino is the LSP and $\chi_{1}$ the next-toLSP. In this case, all superpartner production events at colliders will yield at least two $\chi_{1}$, with each decaying predominantly into a gravitino and a pseudo-Goldstone $G$, with a width of order $10^{-5} \mathrm{eV}$ for $\sqrt{F}=100 \mathrm{TeV}$. Given the scaling $\Gamma\left(\chi_{1}\right) \propto 1 / F^{2}, \chi_{1}$ could therefore decay inside the detector if $\sqrt{F}$ is less than of order $1000 \mathrm{TeV}$. Pair production of $\chi_{1}$ at LEP2 has a cross section of order $0.1 \mathrm{pb}$, leading in this case to events with $4 \mathrm{~b}$ jets and missing energy. We do not know if searches by the LEP experiments would have detected this signal. If not, the generic superpartner production signal at LHC/TeVatron may include $4 b$ jets together with the missing energy. The naturalness argument by itself is not sufficiently tight to prefer $\chi_{1}$ decays inside rather than outside the detector.

Finally, the consistency of our model with the ElectroWeak Precision Tests merits further work; in particular, a non-negligible correction to the T parameter can be induced by values of $\lambda$ in the region of $0.6-0.8$.

It is important to note that there are alternative versions of the NMSSM with extra matter that incorporate both an enhanced Higgs boson mass, perturbative gauge coupling unification and a solution to the $\mu$ problem. The absence of mass parameters in the superpotential may be guaranteed by an $R$ symmetry, that nevertheless allows the interaction $(\kappa / 3) S^{3}$ as well as $\lambda S H_{1} H_{2}$. In order that the $S^{3}$ interaction not substantially reduce the Higgs mass, the weak scale value of $\kappa$ should be less than about 0.1 . However, the form of the renormalization group equations allows $\kappa$ at the unification scale to be close to unity, so this is not a powerful constraint on the theory. What is the form of the $R$ symmetry breaking in the supersymmetry breaking scalar interactions? If $A_{\lambda}$ is the only significant $R$ breaking parameter, and $m_{S}^{2}>0$, then this theory is a perturbation of the model discussed in this paper. On the other hand there is a new minimum for $m_{S}^{2}<0$ that is very different from the one examined here, where the $S^{3}$ interaction prevents runaway behavior for $v_{s}$. There are also models of both the PQ and $\mathrm{R}$ types with large values of the symmetry breaking in the soft scalar interactions, but in these cases there are several more parameters that enter the phenomenology. Nevertheless, these models may be of interest since they may remove the need to tune $m_{S}^{2}$ to small values.

Note added. After completion of this work we became aware of the work of P. Schuster and N. Toro [26] where the NMSSM in the PQ and in the R-symmetric limits is analyzed with special emphasis on the fine tuning issue. We believe that the present work usefully complements Ref. [26] in many different aspects. 


\section{Acknowledgements}

We thank Steve King for useful discussions at the early stages of this work, and in particular for pointing out Ref. [8]. We thank Beate Heinemann, Michelangelo Mangano, and especially Roberto Tenchini for very useful discussions of various searches at the TeVatron, LHC, and LEP. We thank Manuel Drees for pointing out [19]. The work of R.B. was supported in part by the EU under RTN contract MRTN-CT-2004-503369, and by the Humbolt Research Foundation. The work of L.J.H. was supported in part by the NSF grant PHY-04-57315 and by the US Department of Energy under contract DE-AC02-05CH11231.

\section{A 2-loop beta functions}

For the convenience of the reader we give here 2-loop supersymmetric beta functions used to produce results in Section 2. The gauge coupling beta functions are:

$$
\begin{aligned}
\frac{d g_{i}}{d \log \mu}= & \frac{1}{16 \pi^{2}} b_{i} g_{i}^{3}+\frac{1}{\left(16 \pi^{2}\right)^{2}} g_{i}^{3}\left(\sum_{j=1}^{3} b_{i j} g_{j}^{2}-b_{i ; t o p} y_{t}^{2}-b_{i ; \lambda} \lambda^{2}\right), \\
b_{i}= & {\left[\begin{array}{c}
\frac{33}{5}+n_{5} \\
1+n_{5} \\
-3+n_{5}
\end{array}\right], \quad b_{i ; t o p}=\left[\begin{array}{c}
\frac{26}{5} \\
6 \\
4
\end{array}\right], \quad b_{i ; \lambda}=\left[\begin{array}{c}
\frac{6}{5} \\
2 \\
0
\end{array}\right], } \\
b_{i j}= & {\left[\begin{array}{ccc}
\frac{199}{25}+\frac{7}{15} n_{5} & \frac{27}{5}+\frac{9}{5} n_{5} & \frac{88}{5}+\frac{32}{15} n_{5} \\
\frac{9}{5}+\frac{3}{5} n & 25+7 n_{5} & 24 \\
\frac{11}{5}+\frac{4}{15} n_{5} & 9 & 14+\frac{34}{3} n_{5}
\end{array}\right] . }
\end{aligned}
$$

Our result for $b_{i j}$ agrees with [25]. The dependence of $b_{i j}$ on $n_{5}$ as given in [8] is wrong.

The relevant beta functions of $y_{t}$ and of the NMSSM couplings $\lambda, \kappa$ are

$$
\begin{aligned}
& \frac{d y_{t}}{d \log \mu}=\frac{y_{t}}{16 \pi^{2}}\left(6 y_{t}^{2}+\lambda^{2}-\frac{13}{15} g_{1}^{2}-3 g_{2}^{2}-\frac{16}{3} g_{3}^{2}\right)-\frac{y_{t}}{\left(16 \pi^{2}\right)^{2}}\left(22 y_{t}^{4}+3 \lambda^{2} y_{t}^{2}+3 \lambda^{4}+2 \kappa^{2} \lambda^{2}\right), \\
& \frac{d \lambda}{d \log \mu}=\frac{\lambda}{16 \pi^{2}}\left(4 \lambda^{2}+3 y_{t}^{2}+2 \kappa^{2}-g_{1}^{2}-3 g_{2}^{2}\right)-\frac{\lambda}{\left(16 \pi^{2}\right)^{2}}\left(10 \lambda^{4}+9 y_{t}^{2} \lambda^{2}+9 y_{t}^{4}+8 \kappa^{4}+12 \lambda^{2} \kappa^{2}\right), \\
& \frac{d \kappa}{d \log \mu}=\frac{\kappa}{16 \pi^{2}}\left(6 \kappa^{2}+6 \lambda^{2}\right)-\frac{\kappa}{\left(16 \pi^{2}\right)^{2}}\left(24 \kappa^{4}+24 \lambda^{2} \kappa^{2}+12 \lambda^{4}+18 y_{t}^{2} \lambda^{2}\right) .
\end{aligned}
$$

In our analysis we have omitted the two loop contributions of gauge couplings to the running of $y_{t}, \lambda, \kappa$. This is legitimate since $g_{i}$ do not approach non-perturbative values in the UV.

\section{References}

[1] R. Harnik, G. D. Kribs, D. T. Larson and H. Murayama, "The minimal supersymmetric fat Higgs model," Phys. Rev. D 70 (2004) 015002 [arXiv:hep-ph/0311349]. 
[2] R. Barbieri, L. J. Hall, Y. Nomura and V. S. Rychkov, "Supersymmetry without a light Higgs boson," Phys. Rev. D 75 (2007) 035007 arXiv:hep-ph/0607332.

[3] R. Dermisek and J. F. Gunion, "Escaping the large fine tuning and little hierarchy problems in the next to minimal supersymmetric model and $h \rightarrow a a$ decays," Phys. Rev. Lett. 95 (2005) 041801 arXiv:hep-ph/0502105].

[4] S. Chang, P. J. Fox and N. Weiner, "Naturalness and Higgs decays in the MSSM with a singlet," JHEP 0608 (2006) 068 [arXiv:hep-ph/0511250].

[5] R. Dermisek and J. F. Gunion, "A Comparison of Mixed-Higgs Scenarios In the NMSSM and the MSSM," arXiv:0709.2269 [hep-ph].

[6] L. J. Hall and T. Watari, "Electroweak supersymmetry with an approximate U(1)(PQ)," Phys. Rev. D 70, 115001 (2004) arXiv:hep-ph/0405109.

[7] D. J. Miller, R. Nevzorov and P. M. Zerwas, "The Higgs sector of the next-to-minimal supersymmetric standard model," Nucl. Phys. B 681, 3 (2004) arXiv:hep-ph/0304049.

[8] M. Masip, R. Munoz-Tapia and A. Pomarol, "Limits on the mass of the lightest Higgs in supersymmetric models," Phys. Rev. D 57, 5340 (1998) arXiv:hep-ph/9801437.

[9] V. Barger, P. Langacker and G. Shaughnessy, "Collider signatures of singlet extended Higgs sectors," Phys. Rev. D 75, 055013 (2007) arXiv:hep-ph/0611239;

V. Barger, P. Langacker, M. McCaskey, M. J. Ramsey-Musolf and G. Shaughnessy, "LHC Phenomenology of an Extended Standard Model with a Real Scalar Singlet," arXiv:0706.4311 [hep-ph]; and references therein to previous work by the same authors.

[10] M. Dine, N. Seiberg and S. Thomas, "Higgs Physics as a Window Beyond the MSSM (BMSSM)," Phys. Rev. D 76, 095004 (2007) [arXiv:0707.0005 [hep-ph]].

[11] P. Langacker and N. Polonsky, "Uncertainties in coupling constant unification," Phys. Rev. D 47, 4028 (1993) arXiv:hep-ph/9210235.

[12] G. L. Kane, T. T. Wang, B. D. Nelson and L. T. Wang, "Theoretical implications of the LEP Higgs search," Phys. Rev. D 71, 035006 (2005) arXiv:hep-ph/0407001.

[13] M. Drees, "A supersymmetric explanation of the excess of Higgs-like events at LEP," Phys. Rev. D 71 (2005) 115006 [arXiv:hep-ph/0502075].

[14] S. G. Kim, N. Maekawa, A. Matsuzaki, K. Sakurai, A. I. Sanda and T. Yoshikawa, "A Solution for Little Hierarchy Problem and $b \rightarrow s \gamma$," Phys. Rev. D 74 (2006) 115016 arXiv:hepph/0609076.

[15] A. Belyaev, Q. H. Cao, D. Nomura, K. Tobe and C. P. Yuan, "Light MSSM Higgs boson scenario and its test at hadron colliders," arXiv:hep-ph/0609079.

[16] ALEPH Collaboration, "Search for neutral MSSM Higgs bosons at LEP," Eur. Phys. J. C 47, 547 (2006) arXiv:hep-ex/0602042.

[17] The DELPHI Collaboration, "Searches for supersymmetric particles in $e^{+} e^{-}$collisions up to $208 \mathrm{GeV}$ and interpretation of the results within the MSSM," Eur. Phys. J. C 31, 421 (2004) arXiv:hep-ex/0311019; 
The OPAL Collaboration, "Search for chargino and neutralino production at $\sqrt{s}=192-209$ GeV to at LEP," Eur. Phys. J. C 35, 1 (2004) arXiv:hep-ex/0401026;

The L3 Collaboration, "Search for charginos and neutralinos in e+ e- collisions at $\sqrt{s}=189$ GeV," Phys. Lett. B 472, 420 (2000) arXiv:hep-ex/9910007.

[18] ALEPH Collaboration, "Single- and multi-photon production in $e^{+} e^{-}$collisions at $\sqrt{s}$ up to 209 GeV," Eur. Phys. J. C28 (2003) 1;

DELPHI Collaboration, "Photon Events with Missing Energy in $e^{+} e^{-}$collisions at $\sqrt{s}=130$ to 209 GeV", Eur.Phys.J. C38 (2005) 395-411 [arXiv:hep-ex/0406019];

L3 Collaboration, "Single- and Multi-Photon events with Missing Energy in e+e- Collisions at LEP", Phys. Lett. B587 (2004) 16, arXiv:hep-ex/0402002];

OPAL Collaboration, "Photonic Events with Missing Energy in e+e- Collisions at $\sqrt{s}=189$ GeV", Eur. Phys. J. C18 (2000) 253.

[19] H. K. Dreiner, O. Kittel and U. Langenfeld, "Discovery potential of radiative neutralino production at the ILC," Phys. Rev. D 74, 115010 (2006) |arXiv:hep-ph/0610020].

[20] R. Dermisek, J. F. Gunion and B. McElrath, "Probing NMSSM scenarios with minimal finetuning by searching for decays of the Upsilon to a light CP-odd Higgs boson," Phys. Rev. D 76 (2007) 051105 [arXiv:hep-ph/0612031].

[21] F. Wilczek, "Problem Of Strong P And T Invariance In The Presence Of Instantons," Phys. Rev. Lett. 40, 279 (1978).

[22] J. F. Gunion, H. E. Haber, G. L. Kane and S. Dawson, "The Higgs Hunter's Guide," 348pp, Westview Press; New Ed edition (2000).

[23] D. Kreinick, "Recent Results in Bottomonium / Ties to Charmonium," arXiv:0710.5929 [hep$\mathrm{ex}]$.

[24] M. Bastero-Gil, C. Hugonie, S. F. King, D. P. Roy and S. Vempati, "Does LEP prefer the NMSSM?," Phys. Lett. B 489, 359 (2000) arXiv:hep-ph/0006198.

[25] C. D. Carone and H. Murayama, "Phenomenology of Minimal SU(5) Unification with Dynamical Supersymmetry Breaking," Phys. Rev. D 53, 1658 (1996) arXiv:hep-ph/9510219.

[26] P. C. Schuster and N. Toro, "Persistent fine-tuning in supersymmetry and the NMSSM," arXiv:hep-ph/0512189 